\title{
HealthAffairs
}

At the Intersection of Health, Health Care and Policy

Cite this article as:

Sarah Gehlert, Dana Sohmer, Tina Sacks, Charles Mininger, Martha McClintock and Olufunmilayo Olopade

Targeting Health Disparities: A Model Linking Upstream Determinants To Downstream Interventions

Health Affairs, 27, no.2 (2008):339-349

doi: 10.1377/hlthaff.27.2.339

The online version of this article, along with updated information and services, is available at:

http://content.healthaffairs.org/content/27/2/339.full.html

For Reprints, Links \& Permissions:

http://healthaffairs.org/1340_reprints.php

E-mail Alerts : http://content.healthaffairs.org/subscriptions/etoc.dtl

To Subscribe: http://content.healthaffairs.org/subscriptions/online.shtml

Health Affairs is published monthly by Project HOPE at 7500 Old Georgetown Road, Suite 600, Bethesda, MD 20814-6133. Copyright (@ 2008 by Project HOPE - The People-to-People Health Foundation. As provided by United States copyright law (Title 17, U.S. Code), no part of Health Affairs may be reproduced, displayed, or transmitted in any form or by any means, electronic or mechanical, including photocopying or by information storage or retrieval systems, without prior written permission from the Publisher. All rights reserved. 


\section{Targeting Health Disparities: A Model Linking Upstream Determinants To Downstream Interventions}

Knowing about the interaction of societal factors and disease can enable targeted interventions to reduce health disparities.

\section{by Sarah Gehlert, Dana Sohmer, Tina Sacks, Charles Mininger, Martha McClintock, and Olufunmilayo Olopade}

ABSTRACT: Certain social/environmental factors put some groups at extraordinary risk for adverse health outcomes, creating health disparities. We present a downward causal model, originating at the population level and ending at disease, with psychological and behavioral responses linking the two. This approach identifies how specific social environments "get under the skin" to cause disease, illustrated with the disparity in mortality from aggressive premenopausal breast cancer suffered by black women. Broadening our lens to consider the entire chain of causal factors, spanning multiple levels and interacting across the life span, heightens our ability to craft specific interventions to address group differences in health. [Health Affairs 27, no. 2 (2008): 339-349; 10.1377/hlthaff.27.2.339]

$\mathrm{H}$ EALTH DISPARITIES OCCUR BY RACE, ethnicity, sex, socioeconomic status, and sexual orientation, with inequities in screening, incidence, treatment, and mortality across a number of diseases and conditions, including cancer, diabetes, cardiovascular disease, infant mortality, and HIV/AIDS. A black/white gap in mortality from common cancer sites has been noted since 1975, for instance, when cancer data were first collected systematically by race and ethnicity. In 1975, black women experienced 39.2 more deaths per 100,000 population from breast cancer than white women. By 2004, that gap had increased to 44.1 excess deaths per 100,000. During the same time period, breast cancer mortality rates among white women decreased from 31.8 to 23.8 per 100,000. Mortality rates

Sarah Gehlert (sgehlert@uchicago.edu) is the Helen Ross Professor at the School of Social Services Administration, University of Chicago, and director of the Center for Interdisciplinary Health Disparities Research (CIHDR). Dana Sohmer is senior research project professional at the CIHDR. Tina Sacks and Charles Mininger are research assistants there. Martha McClintock is codirector of the CIHDR and the David Lee Shillinglaw Distinguished Service Professor in the university's Department of Psychology. Olufunmilayo Olopade is the Walter L. Palmer Distinguished Service Professor in the Department of Medicine. 
from prostate cancer show a similar pattern through time. While mortality rates for white men decreased between 1975 and 2004 (from 29.1 to 23.4 per 100,000), mortality rates for black men increased (from 55.5 to 56.1 per 100,000). ${ }^{1}$

Black/white disparities in breast and prostate cancer mortality are paralleled by other major diseases among other racial and ethnic groups. Black adults are twice as likely as white adults to be diagnosed with diabetes, while Hispanic and American Indian/Alaska Native adults are 1.9 and 2.6 times more likely than whites to be diagnosed with the disease. Additionally, black adults are 2.2 times more likely than white adults to die from complications of diabetes. ${ }^{2}$

a Federal focus on health disparities. The issue of health disparities received major attention in 1998, with the launch of President Clinton's Racial and Ethnic Health Disparities Initiative and his charge to top public officials to address disparities. Subsequently, reducing health disparities was named as one of the two major goals of Healthy People 2010 and continues to be a focus of federal research and policy interventions. ${ }^{3}$ The Minority Health and Health Disparities Research and Education Act of 2000, for example, created the Center for Minority Health and Health Disparities and charged it to lead the development of a National Institutes of Health (NIH) Strategic Plan for health disparities. Since that time, each institute and center at the NIH has developed its own plan.

Importance of the social environment. Although a host of hereditary and individual behavioral factors are linked to health outcomes, we now understand that social circumstances and environmental factors place minority groups at a distinct disadvantage in health and disease. These groups may be exposed to numerous conditions (such as discrimination and unequal treatment in housing, employment, and medical care) experienced less often by more advantaged groups. As such, societal factors that represent upstream determinants should be included in frameworks for determining population health. In these frameworks, upstream determinants are defined as features of the social environment, such as socioeconomic status and discrimination, that influence individual behavior, disease, and health status. Viewing health disparities through a lens that incorporates social/environmental conditions as upstream factors in multilevel models better allows us to design and implement interventions targeted at levels downstream from those conditions. Policymakers and others can then weigh costs and benefits, to choose the most effective.

- The CIHDR model. In this paper, after reviewing existing models of health determinants, we draw on our own work at the University of Chicago's Center for Interdisciplinary Health Disparities Research (CIHDR) on disparities in breast cancer mortality to illustrate a novel model based on identifying each link in a downward causal chain from the population (social) to the disease (genetic) level. That chain, vertically oriented, starts at the top with race, poverty, disruption, and neighborhood crime; moves to isolation, acquired vigilance, and depression; then to stresshormone dynamics; and finally to cell survival and tumor development. ${ }^{4}$ Although not the only disease-specific model of health determinants, the CIHDR model is 
unique in its ability to demonstrate important links among determinants. ${ }^{5}$ Finally, we review studies that identify links in the causal chain between upstream determinants and health and disease.

\section{Existing Models Of Health Determinants}

Although group differences have been recognized for almost 100 years and have become a public health target in the past decade, understanding how they develop and are sustained has lagged behind their recognition. Investigators generally agree that health disparities are determined by many factors and that influences occur at group and individual levels. Early models, although they included grouplevel risk factors, focused primarily on individual-level factors. ${ }^{6}$ Awareness of the number and influence of group-level factors increased through time, and frameworks began to acknowledge the impact of the social and physical environments on individual behavior and biology.

An emphasis on the complex interactions between levels of health determinants allowed a focus on population as well as individual health outcomes and provided a valuable tool for understanding how group differences in health develop. In 2000, George Kaplan and colleagues proposed a framework with levels representing social and economic policy, institutions, neighborhoods and communities, living conditions, social relationships, individual risk factors, genetic and constitutional factors, and pathophysiological pathways that interact with the environment throughout the life course to affect both individual and population health. ${ }^{8}$ They held that "it is highly unlikely that the triad of genomics, bioinformatics, and biomedicine, and their focus on molecular etiologic forces located within the individual, will explain very much of the heterogeneity in health and disease among social groups, places and times," and they suggested an approach that "bridges various levels of explanation."

\section{The CIHDR Downward Causal Model}

Downward causation. Most existing frameworks assume a hierarchical arrangement of levels, with the primary cause arising from the smallest level of organization-namely, the genome, which interacts with the cellular environment within the body to cause disease. In this framework, social and behavioral factors are implicitly viewed as less powerful "risk" factors, almost to the point of being considered outcomes influenced or shaped by genes. We, on the other hand, emphasize downward causation, in which upstream determinants at the social and environmental levels influence and regulate events at lower levels of organization (that is, from individual behavior and physiology to the cellular and genetic interactions of health and disease).

The notion of downward causation was first articulated by Donald Campbell in 1974 to describe how higher organizational levels influence lower levels in hierarchically organized biological systems, such that "all processes at the lower levels 
of a hierarchy are restrained by and act in conformity at the laws of the higher levels." Intermediate-level factors can also be described with reference to laws at a higher level of organization.

Although this language is used to describe the causal role of social, behavioral, and biological factors on health outcomes, it is important to acknowledge that feedback also occurs from lower to higher levels, with genetic and biological factors influencing phenomena above them in traditional hierarchies. ${ }^{10}$ Examples abound with the rapid increase in technology enabling the sequencing of heritable variations within genes. Variation in base sequences of a gene can alter how well it is expressed, causing heritable genetic variation among people and higher prevalence of a disease in subpopulations. For example, phenylketonuria (PKU) is caused by heritable mutations (that is, differences in base sequences) within the PAH gene and results in mental retardation. It is more common among Asians and whites, especially of Turkish descent, but rare among blacks.

Interactions among genes, their products, and the environment that bring a phenotype into being (for example, mental retardation or health) were termed "epigenetics" by Conrad Waddington well before DNA was discovered or the mechanisms of gene function understood. ${ }^{11}$ Today, the term refers to "inheritance systems through which non-DNA variations can be transmitted." 12 In other words, without changing the heritable genes that will be passed on to the next generation, we know of mechanisms within cells and their nuclei through which the environment can change the way DNA and cells function. These changes can then be maintained and transmitted through cell-division cycle after cell-division cycle within a person's lifetime, if not transmitted between generations.

The upstream environments that change the way DNA and cells throughout the body function include not only a person's physiology, but also his or her behavior and physical and social environments. Such high-level epigenetic triggers are central to the primarily downward causal model of breast cancer developed by CIHDR investigators, in which multiple environments at different levels interact to regulate gene expression. ${ }^{13}$

Breast cancer among black women. The CIHDR is one of eight centers funded in 2003 through the NIH's Centers for Population Health and Health Disparities (CPHHD) initiative. The four CIHDR projects are led by social, behavioral, and biological scientists working together with community stakeholders. The central question that unites them is why, despite the fact that white women are more likely to develop breast cancer, black women are more likely to die from it. Seventy to eighty percent of human breast cancers are due to acquired rather than inherited mutations of breast cancer genes, and breast cancer develops after a series of complex genetic interactions. ${ }^{14}$ The four mutually informative, interdependent CIHDR projects aim to determine the causal links through which these genetic mechanisms are regulated by the social environment. The projects share a model of downward causation, from the social environment to the survival of malignant cells, which re- 
sults in the development of tumors (that is, the accumulation of malignant cells) in breast cancer.

Animal models. Two projects use animal models that mimic human disease to identify pathways by which social and psychological factors "get under the skin" to influence disease. This greatly adds to the CIHDR's understanding of the determinants of health disparities in humans, in two major ways. First, it allows social conditions to be manipulated using experiments (and psychological reactions, behavior, and biological processes to be measured). Second, it affords a perspective on gene/environment interactions throughout the entire life cycle.

CIHDR investigators have found that rodents that are socially isolated from the time of weaning become vigilant and develop larger spontaneous mammary gland tumors at a much earlier age than their group-housed peers. ${ }^{15}$ Tumor development is accompanied by an increase in stress-hormone response to an acute stressor, which raises the possibility that increased stress-hormone signaling may mediate an increase in tumor growth in isolated animals.

Human models. Two CIHDR projects follow the same group of 230 black women newly diagnosed with breast cancer living in predominantly black neighborhoods of Chicago, one analyzing the characteristics of their breast tumors and the other their social situations and psychosocial functioning. The model defines race in terms of the social-environmental factors that might determine which women will experience epigenetic changes that produce sporadic breast cancers. ${ }^{16}$

Study results. Our work with animal models informs the CIHDR model of downward causation from social environmental conditions to psychosocial factors, biological responses, and breast tumor development among newly diagnosed black women living in Chicago. Using information gathered from in-home interviews, investigations of the built environment, and publicly available data geocoded to the women's addresses, we have been able to demonstrate a pathway with significant associations between levels, from the community to inside the nucleus of the cell, in which dilapidated housing, crimes, and generally fractured communities engender isolation and depression, and in so doing, alter stress-hormone response. ${ }^{17}$ Investigators have identified stress-hormone (glucocorticoid) receptors that are activated by social isolation that further downstream activate biochemical pathways known to increase tumor cell survival. Expression of this biochemical pathway occurs when a hormone travels across the cell membrane and binds with the stress-hormone receptor complex, which goes into the nucleus of the cell and causes the gene to express the pathway, thus creating the "soil" in which cancer grows.

\section{Established Linkages Between Levels Of Health Determinants}

A number of studies have demonstrated major links between levels of health determinants. These allow us to understand how intervening at one level will affect factors downstream from that level and craft interventions to reduce disparities. 


\section{"Social isolation is important for understanding health disparities, because of its links to numerous health outcomes."}

From the neighborhood/community level downstream. Neighborhoods and communities have always been important upstream determinants of health outcomes. Empirical studies have documented an association between neighborhood context and health in areas such as mortality, low birthweight, depression, cancer, and cardiovascular disease. ${ }^{18}$ The degree to which people engage, form relationships, and leverage resources can be traced to surrounding social structures, how people fit into these structures, and the economic realities that they present. ${ }^{19}$ Variation in neighborhood organization may promote or impede social interaction-a critical determinant of health status. ${ }^{20}$

Social forces such as discrimination, segregation, and urban inequality have a direct impact on neighborhoods and their uses and further affect social interaction by shaping how people perceive their circumstances, influencing where and with whom they live, and shaping available resources. ${ }^{21}$ These conditions can engender social isolation by limiting the number and types of relationships people hold as well as the frequency of their interactions. Social isolation is important for understanding health disparities, because of its links to numerous health outcomes, including all-cause mortality. ${ }^{22}$

The quality and content of the built environment of neighborhoods, defined as the buildings, spaces, and products created by people, have a profound effect on health outcomes. For example, deteriorated infrastructures and overall design of buildings have been shown to affect the formation of relationships and maintenance of collective efficacy. ${ }^{23}$ Neighborhoods with fewer signs of occupation, fences, and higher speed limits are more likely than others to be burglarized. ${ }^{24}$ These issues may cause residents to retreat into their homes, thus limiting interaction and increasing feelings of loneliness. In addition, attempts to deal with threats may deplete people's physical or psychological resources over time.

Linking the psychosocial level to physiology. Despite evidence that socialenvironmental conditions are associated with health outcomes, we are only beginning to understand the causal pathways through which social context contributes to health disparities. The CIHDR's downward causal model, for example, describes a particular multifaceted psychological state linked to the physiological stress system through which social circumstances may affect a person's risk for breast cancer. No doubt other pathways will be identified for this and other diseases.

One way that the social environment affects health is by challenging people's ability to respond to environmental stimuli and changing their psychological states. The link between psychological states and disease outcomes has been explored in terms of the physiological changes that occur with variations in emotional or behavioral states. ${ }^{25}$ For example, depression is characterized by several 
biological distinctions, including changes in autonomic nervous system activities, as evidenced by increased sympathetic nervous system activation and decreased parasympathetic nervous system tone. In depression, the hypothalamic-pituitaryadrenal (HPA) axis maintains low levels of stress hormones (glucocorticoids), yet heightened release in response to a stressor and disrupted catecholamine cycles are manifested by increased release of epinephrine (adrenaline). ${ }^{26}$ Dysregulation of physiological processes can lead to other changes, such as suppressed immune function, decreased blood flow to the digestive system, and inhibited growth hormones, which can ultimately lead to poor health outcomes. ${ }^{27}$

Another way in which social/environmental conditions influence psychological states and subsequent physiological stress reactions is through social isolation and the loneliness it engenders. Loneliness has been linked to various cardiac activations, decreased cellular immune function, and increased release of stress hormones. ${ }^{28}$ Disrupted social connections may hamper a person's ability to cope with social/environmental stressors, which in turn can disregulate physiological processes and affect disease outcomes downstream.

- Physiological mechanisms and pathways to disease. When a person is challenged by the environment, a large set of interacting physiological systems must accommodate each other, changing in different ways to maintain function - the dynamic response of a complex system first termed allostasis by Peter Sterling and Joseph Eyer. ${ }^{29}$ Although often beneficial in the short term, maintaining allostasis over the long term creates a load that can result in disease. Social challenges can also create allostatic load, particularly when demands exceed supports. ${ }^{30}$ With repeated cycles of allostasis in response to enduring challenges, allostatic load is manifested by the wear and tear that the body endures and its inability to turn the system on and off efficiently.

People vary in their ability to cope with environmental challenges, based on genetic, developmental, and experiential factors, including the long-term effect of early-life stress that may predispose people to overreact physiologically and behaviorally. Arline Geronimus and colleagues found that blacks of all ages have higher allostatic load scores than whites that were not entirely explained by individual socioeconomic status, which suggests that the cumulative impact of social and economic adversity can profoundly affect health. ${ }^{31}$ For example, blacks ages 18-24 were almost 50 percent more likely to have a higher allostatic load score than whites of the same age. By ages 55-64, the black-white relative odds ratio rose to 2.31, indicating that blacks were more than twice as likely as whites to show the physiological effects of high-effort coping.

Minority groups, including those of moderate and upper income, often face multiple environmental and social risks. The Geronimus study highlights the need to contextualize the experiences of minorities to include more nuanced measures of socioeconomic status, as well as other disease risk factors. Given that social arrangements are likely to have a synergistic effect on a person's stress response, in- 
terventions to address racial disparities in health should consider the interaction between individual susceptibility to stress and the environmental conditions that may lead to disease expression.

\section{Downstream Interventions}

An advantage of clearly articulated, disease-specific models of health disparities with demonstrated links between levels is that they allow us to predict the impact of interventions aimed at one level on factors downstream from that level. ${ }^{32}$ Although this approach is in its infancy, designing and targeting interventions based on empirically established relationships has the potential to save resources and heighten the effectiveness of interventions in reducing health disparities. Interventions that focus on one level of determinants without considering others may affect individual outcomes while making a less-than-desired dent in group health differences.

Decreasing social isolation. As an example, we know that black women have markedly higher breast cancer mortality rates than white women. They also are more likely to experience the so-called triple-negative cancers (those lacking receptors for three hormones-namely, estrogen, progesterone, and HER2/neu) that develop at a younger age, often before menopause, and are more lethal and aggressive than other cancers. ${ }^{33}$ We hypothesize that a number of upstream factors ultimately produce or at least increase the risk for triple-negative cancers. Identifying these upstream factors will help to target interventions.

In the CIHDR investigations, neighborhood characteristics that discourage social interaction may be linked to hormone profiles that have the potential to produce triple-negative tumors. If so, interventions to increase collective efficacy and improve neighborhood safety should reduce breast cancer mortality by disrupting the link between isolation and loneliness and gene expression changes. In her discussion of the effects of urban renewal on inner-city neighborhoods, Mindy Fullilove suggests offering places for exchange in neighborhoods, not unlike the settlement houses of the early 1900s. ${ }^{34}$

Another avenue for decreasing social isolation in neighborhoods targets the vacant buildings that foster crime and negatively affect the formation and maintenance of social relationships. A number of municipalities have begun efforts to ensure that landlords maintain properties by levying fines and financial disincentives. Examples include Cleveland's Housing Court and Chicago's Troubled Building Initiative. The latter placed twenty properties in receivership during its first three years of operation. ${ }^{35}$

Early detection. Factors that interact with cancer type also are potential points of intervention. Because triple-negative cancers grow fast, detecting them at an early stage is a key to survival. While the gap between white and black women in breast cancer screening has narrowed, it remains the case that facilities in predominantly black communities are (1) less likely to offer timely breast cancer screening 
using state-of-the-art techniques, and (2) more likely to misread mammograms than are clinics in more-affluent areas. ${ }^{36}$ Ensuring that inner-city health facilities have up-to-date, well-maintained equipment and that mammographers have access to continuing training and opportunities for consultation should help reduce breast cancer mortality among black women.

- Including social indicators with clinical information. Individual risk profiles that capture multiple components of people's social circumstances as well as the subclinical indicators of the physiological effects that flow from these circumstances may serve as effective primary and secondary prevention strategies and help improve individual and population health outcomes. The cross-level effects of individual and neighborhood socioeconomic status, for example, are significantly related to mortality from a number of diseases. ${ }^{37}$ Including this type of cross-level information with clinical information may help providers to design individualized prevention programs and clinicians to choose treatment strategies targeted to patients' specific needs.

Community partnerships. Understanding the nature of upstream determinants is best achieved through partnerships with community stakeholders. Amy Schulz and colleagues, for example, worked with community practitioners and stakeholders in Detroit to develop a model of the pathways through which the social and physical environments influence racial and socioeconomic disparities in cardiovascular disease. ${ }^{38}$

Community-based participatory research approaches, which combine research and social change, have also proved useful in devising and testing the effectiveness of interventions, in areas such as church-based diabetes prevention. ${ }^{39}$ Involving stakeholders likewise helps ensure that interventions are "owned" by community members and thus more likely to be sustained through time.

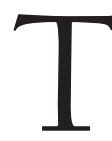

he Determinants of health Disparities are multiple, and none operates in isolation. We are just beginning to understand how factors at different levels interact to influence population health outcomes. Considering these interactions will allow us to choose points of intervention, knowing how the resulting change will affect downstream factors. This approach is economical because it in essence allows us to produce multilevel changes. It greatly heightens our chances of devising interventions that truly affect group differences in health.

Reports of the nation's progress in addressing health disparities are less than encouraging, and much remains to be done to meet the goals of Healthy People 2010. ${ }^{40}$ Carefully targeted interventions based on well-articulated, diseasespecific models, such as the CIHDR's downward causal model of breast cancer, hold promise for speeding our progress toward achieving those goals.

The authors thank the National Institute of Environmental Health Sciences and the National Cancer Institute for their support through Grant no. P50ES012382 to Sarah Gehlert.

HEALTH AFFAIRS Volume 27, Number 2 


\section{NOTES}

1. L.A.G. Ries et al., eds., SEER Cancer Statistics Review, 1975-2004, http://seer.cancer.gov/csr/1975_2004/results merged/topic_annualrates.pdf (accessed 2 August 2007).

2. Centers for Disease Control and Prevention, "Total Prevalence of Diabetes by Race/Ethnicity among People Aged Twenty Years or Older, United States, 2002," http://www.cdc.gov/diabetes/pubs/estimates .htm \#prev4 (accessed 2 August 2007).

3. U.S. Department of Health and Human Services, Healthy People 2010: Understanding and Improving Health (Washington: U.S. Government Printing Office, 2000); and G. Thompson, F. Mitchell, and M. Williams, eds., Examining the Health Disparities Research Plan of the National Institutes of Health: Unfinished Business (Washing ton: National Academies Press, 2006).

4. For a graphic depicting the CIHDR model, go to http://content.healthaffairs.org/cgi/content/full/27/2/339/ $\mathrm{DCl}$.

5. E. Brunner and M. Marmot, "Social Organization, Stress, and Health," in Social Determinants of Health, ed. M. Marmot and R. Wilkinson (Oxford: Oxford University Press, 2000), 17-43; and S. Gehlert et al., "Cells to Society: Overcoming Health Disparities," Interim Progress Report (Washington: National Cancer Institute, November 2007).

6. M. Lalonde, A New Perspective on the Health of Canadians: A Working Document (Ottawa: Minister of Supply and Services Canada, 1974).

7. M. Von Korff et al., "Multi-Level Analysis in Epidemiologic Research on Health Behaviors and Outcomes," American Journal of Epidemiology 135, no. 10 (1992): 1077-1082; and R.G. Evans and G.L. Stoddart, "Producing Health, Consuming Health Care," Social Science and Medicine 31, no. 12 (1990): 1347-1363.

8. G. Kaplan, S.A. Everson, and J.W. Lynch, "The Contribution of Social and Behavioral Research to an Understanding of the Distribution of Disease: A Multilevel Approach," in Promoting Health: Intervention Strategies from Social and Behavioral Research, ed. B.D. Smedley and R. Sampson (Washington: National Academies Press, 2000), 42.

9. D. Campbell, "Downward Causation' in Hierarchically Organized Biological Systems," in Studies in the Philosophy of Biology: Reductionism and Related Problems, ed. F. Ayala and T. Dobzhansky (Berkeley and Los Angeles: University of California Press, 1974), 180.

10. L. Hernandez and D. Blazer, eds., Genes, Behavior, and the Social Environment: Moving Beyond the Nature/Nurture Debate (Washington: National Academies Press, 2006).

11. C.H. Waddington, "The Epigenotype," Endeavor 1, no. 1 (1942): 18-20.

12. E. Jablonka and M.J. Lamb, "The Changing Concept of Epigenetics," Annals of the New York Academy of Sciences 981 (2002): 82-96.

13. M.K. McClintock et al., "Mammary Cancer and Social Interactions: Identifying Multiple Environments That Regulate Gene Expression throughout the Life Span," Journals of Gerontology, Series B-Psychological Sciences and Social Sciences, 60, Special Issue 1 (2005): 32-41.

14. R. Wooster and B.L. Weber, "Breast and Ovarian Cancer," New England Journal of Medicine 348, no. 23 (2003) 2339-2347.

15. G.L. Hermes et al., "Social Isolation and the Inflammatory Response: Sex Differences in the Enduring Effects of a Prior Stressor," American Journal of Physiology 290, no. 2 (2006): R273-R282.

16. McClintock et al., "Mammary Cancer and Social Interactions."

17. Gehlert et al., "Cells to Society."

18. I.H. Yen and G.A. Kaplan, "Neighborhood Social Environment and Risk of Death: Multilevel Evidence from the Alameda County Study," American Journal of Epidemiology 149, no. 10 (1999): 898-907; J.D. Morenoff, "Neighborhood Mechanisms and the Spatial Dynamics of Birth Weight," American Journal of Sociology 108, no. 5 (2003): 976-1017; C.E. Ross, "Neighborhood Disadvantage and Adult Depression," Journal of Health and Social Behavior 41, no. 2 (2000): 177-187; C.D. Jenkins, "Social Environment and Cancer Mortality in Men," New England Journal of Medicine 308, no. 7 (1983): 395-398; and A.V. Diez-Roux et al., "Neighborhood Environments and Coronary Heart Disease: A Multilevel Analysis," American Journal of Epidemiology 146, no. 1 (1997) $48-63$.

19. L.I. Pearlin, "The Sociological Study of Stress," Journal of Health and Social Behavior 30, no. 3 (1989): 241-256.

20. J.S. House, K.R. Landis, and D. Umberson, "Social Relationships and Health," Science 241, no. 4865 (1988): 540-545.

21. D.S. Massey and N.A. Denton, American Apartheid: Segregation and the Making of the Underclass (Cambridge, 
Mass.: Harvard University Press, 1993); and W.J. Wilson, The Truly Disadvantaged: The Inner City, the Underclass, and Public Policy (Chicago: University of Chicago Press, 1987).

22. L.F. Berkman and S.L. Syme, "Social Networks, Host Resistance, and Mortality: A Nine-Year Follow-up Study of Alameda County Residents," American Journal of Epidemiology 109, no. 2 (1979): 186-204

23. R.J. Sampson, S.W. Raudenbush, and F. Earls, "Neighborhoods and Violent Crime: A Multilevel Study of Collective Efficacy," Science 277, no. 5328 (1997): 918-924; G. Rand, "Crime and Environment: A Review of the Literature and Its Implications for Urban Architecture and Planning," Journal of Architectural Planning and Research 1, no. 1 (1984): 3-19; and O. Newman, Defensible Space: Crime Prevention through Urban Design (New York: Macmillan, 1972).

24. Rand, "Crime and Environment."

25. L.D. Kubzansky and I. Kawachi, "Affective States and Health," in Social Epidemiology, ed. L.F. Berkman and I. Kawachi (New York: Oxford University Press, 2000), 213-24l.

26. Institute of Medicine, Health and Behavior: The Interplay of Biological, Behavioral, and Societal Influences (Washington: National Academies Press, 2001); and D.F. Swaab, A.M. Bao, and P.J. Lucassen, "The Stress System in the Human Brain in Depression and Neurodegeneration," Ageing Research Reviews 4, no. 2 (2005): 141-194.

27. H.D. Sesso et al., "Depression and the Risk of Coronary Heart Disease in the Normative Aging Study," American Journal of Cardiology 82, no. 7 (1998): 851-856.

28. J.K. Kiecolt-Glaser et al., "Psychosocial Modifiers of Immunocompetence in Medical Students," Psychosomatic Medicine 46, no. 1 (1984): 7-14; and J.T. Cacioppo et al., "Lonely Traits and Concomitant Physiological Processes: The MacArthur Social Neuroscience Studies," International Journal of Psychophysiology 35, no. 2/3 (2000): 143-154.

29. P. Sterling and J. Eyer, "Allostasis: A New Paradigm to Explain Arousal Pathology," in Handbook of Life Stress, Cognition, and Health, ed. S. Fisher and J. Reason (New York: John Wiley and Sons, 1988), 629-649.

30. B.S. McEwen and J.C. Wingfield, "The Concept of Allostasis in Biology and Biomedicine," Hormones and Behavior 43, no. 1 (2003): 2-15.

31. A.T. Geronimus et al., "Weathering' and Age Patterns of Allostatic Load Scores among Blacks and Whites in the United States," American Journal of Public Health 96, no. 5 (2006): 826-833.

32. Gehlert et al., "Cells to Society."

33. L.A. Carey et al., "Race, Breast Cancer Subtypes, and Survival in the Carolina Breast Cancer Study," Journal of the American Medical Association 295, no. 21 (2006): 2492-2502; and S. Reynolds, "Triple-Negative Breast Cancer Disproportionately Affects African American and Hispanic Women," Cancer Bulletin, 2007, pp. 7-8, http://www.cancer.gov/ncicancerbulletin/NCI_Cancer_Bulletin_072407.pdf (accessed 8 August 2007).

34. M. Fullilove, Root Shock: How Tearing Up City Neighborhoods Hurts America, and What We Can Do about It (New York: Ballantine Press, 2004).

35. W.D. Keating, "Preserving Properties on the Edge: Rapid Recycling of Distressed and Abandoned Properties" (Unpublished manuscript, Harvard University, March 2007).

36. J.G. Elmore et al., "Racial Inequities in the Timing of Breast Cancer Detection, Diagnosis, and Initiation of Treatment," Medical Care 43, no. 2 (2005): 141-148; J. Hirschman, S. Whitman, and D. Ansell, "The Black:White Disparity in Breast Cancer Mortality: The Example of Chicago," Cancer Causes and Control 18, no. 3 (2007): 323-333; and N. Breen et al., "Progress in Cancer Screening over a Decade: Results of Cancer Screening from the 1987, 1992, and 1998 National Health Interview Surveys," Journal of the National Cancer Institute 93, no. 22 (2001): 1704-1713.

37. M. Winkleby, C. Cubbin, and D. Ahn, "Effect of Cross-Level Interaction between Individual and Neigh borhood Socioeconomic Status on Adult Mortality Rates," American Journal of Public Health 96, no. 12 (2006): 2145-2153.

38. A.J. Schulz et al., "Social and Physical Environments and Disparities in Risk for Cardiovascular Disease: The Healthy Environments Partnership Conceptual Model," Environmental Health Perspectives 113, no. 12 (2005): 1817-1825.

39. J.M. Boltri et al., "Developing a Church-Based Diabetes Prevention Program with African Americans: Focus Group Findings," Diabetes Education 32, no. 6 (2006): 901-909.

40. Agency for Healthcare Research and Quality, National Healthcare Disparities Report, 2006, http://www.ahrq .gov/qual/nhdr06/nhdr06.htm (accessed 3 December 2007). 